\title{
„Der Mensch, der Handball spielt, braucht den Ball, den Kontakt und die Gemeinschaft" - Veränderungen im Sportangebot durch die COVID-19 Pandemie und deren Bedeutung für Sportvereine
}

\author{
The Person Who Plays Handball Needs the Ball, the Contact and \\ the Community - Changes in Sport Club Activities Due to the \\ COVID-19 Pandemic and its Significance for Sports Clubs
}

Autoren

Malte Kehl' ${ }^{1}$, Helmut Strobl², Susanne Tittlbach², Julika Loss ${ }^{3}$

Institute

1 Medizinische Soziologie, Institut für Epidemiologie und Präventivmedizin, Universität Regensburg, Regensburg, Deutschland

2 Institut für Sportwissenschaft, Universität Bayreuth, Bayreuth, Deutschland

3 Abteilung für Epidemiologie und Gesundheitsmonitoring, Robert Koch Institut, Berlin, Deutschland

\section{Schlüsselwörter}

Corona, Gesundheitsförderung, Digitalisierung, Experteninterviews, Sportvereine, Sport

\section{Key words}

Corona, health promotion, digitization, expert interviews, sport clubs, sport

online publiziert $\quad 28.1 .2021$

\section{Bibliografie}

Gesundheitswesen 2021; 83: 159-165

DOI 10.1055/a-1341-1609

ISSN 0949-7013

(c) 2021. Thieme. All rights reserved. Georg Thieme Verlag KG, Rüdigerstraße 14, 70469 Stuttgart, Germany

\section{Korrespondenzadresse}

Malte Kehl

Medizinische Soziologie

Institut für Epidemiologie und

Präventivmedizin Fakultät für Medizin

Universität Regensburg

Dr.-Gessler-Straße 17

93051 Regensburg

Deutschland

Malte.Kehl@klinik.uni-regensburg.de

\section{ZUSAMMENFASSUNG}

Ziel der Studie Sportvereine spielen eine wichtige Rolle für die Bewegungs- und damit auch die Gesundheitsförderung in Deutschland. Die vorliegende Studie befasst sich vor dem Hintergrund der Corona-bedingten Einschränkungen mit den Veränderungen in der Angebotsstruktur und deren Bedeutung für die Sportvereine.

Methode Es wurden 15 Experteninterviews mit Vertretern und Vertreterinnen bayerischer Sportvereine geführt. Die Daten wurden transkribiert und mittels thematischer Analyse induktiv ausgewertet. Ergebnisse Von den Vereinen wurden umfangreiche Aktivitäten entwickelt, um den Mitgliedern auch unter den Rahmenbedingungen der COVID-19-Pandemie Möglichkeiten für sportliche Aktivitäten zu bieten. Die Vereinsmitglieder hätten dabei mit viel Verständnis auf die Veränderungen und Einschränkungen im Angebot reagiert. Die Nutzung der neu geschaffenen digitalen Angebote durch die Mitglieder scheint sich von der Nutzung der realen Kurse zu unterscheiden. Das längerfristige Potenzial der digitalen Angebote wird jedoch weitgehend skeptisch betrachtet. Insbesondere der im Sport normalerweise immanente Gemeinschaftsaspekt würde den Teilnehmenden an digitalen Formaten fehlen.

Schlussfolgerungen Die Anstrengungen der Sportvereine, ein geregeltes Bewegungsprogramm auch unter den Rahmenbedingungen einer Pandemie bereit zu stellen, betont deren Bedeutung für Prävention und Gesundheitsförderung. Aus PublicHealth-Sicht bieten die Veränderungen in der Angebotsstruktur darüber hinaus das Potenzial, auch unter normalisierten Rahmenbedingungen beibehalten zu werden. Hierzu sind aber weiterführende Forschungsarbeiten notwendig.

\footnotetext{
ABSTRACT

Aim Sports clubs play an important role in the promotion of physical activity and health in Germany. Against the background of restrictions due to the COVID-19 pandemic, the present study explores the changes in sport activities and their significance for sports clubs.
} 
Methods We conducted 15 expert interviews with representatives of Bavarian sports clubs. The data were transcribed and evaluated inductively by means of thematic analysis.

Results The clubs developed extensive activities to provide members with opportunities for sport and exercise even under the conditions of the COVID-19 pandemic. In general, the club members were understanding and positive towards the changes in the clubs. The participation in the newly created digital offers by the members seemed to differ from those in real courses. Nevertheless, the inter- viewees were skeptical about the long-term potential of digital sports offerings. Participants could miss the personal contact, the collective experience and team spirit when meeting only virtually. Conclusions The efforts of the sports clubs to provide regular opportunities for sport and exercise during the COVID-19-pandemic underlines their importance for prevention and health promotion. From a public health perspective, changes in the offered activities may outlast the pandemic. However, further research is necessary.

\section{Einleitung}

Mit über 27,5 Mio. Mitgliedern, organisiert in fast 90000 Vereinen, repräsentieren Sportvereine einen elementaren Bestandteil des Breitensports in Deutschland [1]. Durch ihr vielfältiges Bewegungs- und Sportangebot erfüllen sie dabei wichtige Funktionen aus der Perspektive der öffentlichen Gesundheitsförderung (Public Health) [2]. Traditionell stehen in den Sportvereinen klassische wettkampforientierte Sportarten im Mittelpunkt. Gesellschaftliche Veränderungen wie der demografische Wandel führen jedoch zu einer Neuausrichtung bzw. Ausdifferenzierung des Angebots in den Vereinen [3]. Beispielsweise gewinnen Sportangebote mit spezifischem Gesundheitsbezug in den Vereinen zunehmend an Bedeutung. Bereits knapp jeder dritte Verein hat gesundheitssportliche Angebote, und etwa $10 \%$ der deutschen Sportvereine verfügen über Präventions- und Rehabilitationskurse [4].

Entsprechend vielfältig sind die positiven Gesundheitserwartungen, die mit sportlichen Aktivitäten im Verein verknüpft sind. Die Wirkung auf physische Ressourcen wie Kraft, Ausdauer und Koordination sowie psychische Ressourcen wie Stimmung und Wohlbefinden kann bei einer entsprechenden maßvollen und zielgerichteten Ausgestaltung der sportlichen Aktivitäten als unstrittig angesehen werden [5]. Somit kann die aktive Mitgliedschaft im Sportverein dazu beitragen, den unerwünschten Folgen eingeschränkter oder auch fehlender Bewegungserfahrungen entgegenzuwirken [6, 7]. Zudem können gemeinsame sportlichen Aktivitäten ein Zugehörigkeitsgefühl vermitteln sowie Menschen unterschiedlicher Herkunft und unterschiedlichen Alters zusammenführen $[4,8,9]$. Somit bieten Sportvereine bei einer entsprechend methodisch-didaktischen Gestaltung des Trainings das Potenzial, zur Gesundheitsförderung bei verschiedenen Zielgruppen beizutragen. Sie sind darum ein wichtiger Partner bei Projekten der gemeindenahen Gesundheitsförderung (vgl. z. B. [10,11]).

Die zunehmende Verbreitung des Coronavirus (SARS-CoV-2) und die damit einhergehenden Eindämmungsmaßnahmen hatten jedoch auch auf die Sportvereine umfangreiche Auswirkungen. In Deutschland wurden strenge Kontaktbeschränkungen eingeführt, die den Aufenthalt im Freien nur alleine oder in Anwesenheit von Personen des gleichen Hausstands gestatteten [12], zudem wurde das öffentliche Leben weitgehend stillgelegt. Davon betroffen war auch der Betrieb öffentlicher und privater Sportanlagen, darunter Sportstätten und Schwimmbäder, deren Schließung Mitte März 2020 angeordnet wurde [13]. Erst Mitte Mai wurde die schrittweise Wiederöffnung von Angeboten im Breitensport gestattet, jedoch unter strengen Vorgaben zur Einhaltung der Abstandsbestimmungen [14]. Ein geregelter Trainingsbetrieb und der damit verbundene soziale Austausch der Mitglieder im Verein war also für lange Zeit nicht möglich. Auch gegenwärtig ist Breitensport nur unter Einhaltung strenger Hygienekonzepte möglich und die aktuellen Pandemie-Entwicklungen lassen befürchten, dass bestehende Einschränkungen sogar noch weiter verschärft werden können.

Vor diesem Hintergrund befasst sich die vorliegende Studie mit den Konsequenzen, die sich daraus für den Vereinsbetrieb und für die Angebotsstruktur der Vereine ergeben haben. Folgende Fragestellungen stehen im Mittelpunkt:

- Welche Veränderungen ergaben sich aufgrund der Corona-bedingten Einschränkungen für das Bewegungs- und Sportangebot der Vereine?

- Wie haben die Mitglieder auf das veränderte Bewegungs- und Sportangebot reagiert?

- Wie gehen die Vereine mittel- und langfristig mit den Corona-bedingten Einschränkungen um?

\section{Methodik}

Die Studie fand im Rahmen des BMBF-geförderten Forschungsprojektes ACTION for men (A4M) statt, als Teil des Forschungsverbundes Capital4Health. A4M untersucht, inwieweit Akteure auf kommunaler Ebene, v. a. Sportvereine, in die Planung und Implementierung von lokalen Maßnahmen zur Bewegungsförderung eingebunden werden können. Für die hier vorgestellte Erhebung wurde ein qualitatives Studiendesign gewählt. Es fanden 15 leitfadengestützte Experteninterviews [15] mit Vertretungen aus der Vorstandschaft $(n=9)$ sowie Mitarbeitenden $(n=6)$ in 15 Sportvereinen statt (14 Männer, 1 Frau). Die Vereine wurden in 2 bayerischen Regierungsbezirken rekrutiert. So wurde sichergestellt, dass die Vereine von einheitlichen landesweiten Einschränkungsmaßnahmen betroffen waren. Gleichzeitig sollte ein möglichst breites Spektrum von Sportangeboten (von reinen Einsparten-Vereinen $(n=7)$ wie z. B. Skiverein oder Schwimmverein über Mehrspartenvereine $(n=7)$ mit zertifizierten Gesundheitssportkursen bis hin zu Kampfsportvereinen) abgedeckt werden. Die Rekrutierung der Interviewpartner erfolgte durch persönliche Kontakte des Forschungsteams sowie die direkte Kontaktaufnahme mit lokalen Sportvereinen. Es wurden 18 Sportvereine angesprochen, 15 sagten die Teilnahme zu. Interessierte Personen erhielten ein Informationsblatt und willigten schriftlich in die Studienteilnahme ein. Sie wurden weitergehend informiert, dass die Studienteilnahme zu jedem Zeitpunkt und ohne Angabe von Gründen widerrufen werden kann. Für A4M liegt ein positives Votum der Ethikkommission der Universität Bayreuth vor (Az. O 1305/1 - GB). 
Der Interviewleitfaden adressierte folgende 3 Themenbereiche:

a) Veränderungen im Sportangebot der Vereine.

b) Resonanz der Vereinsmitglieder auf das veränderte Sportangebot.

c) (Langfristige) Potenziale und Grenzen der Veränderungen im Sportangebot.

Der Interviewleitfaden wurde im Rahmen eines Pre-Tests auf Verständlichkeit hin überprüft und anschließend geringfügig adaptiert. Die Interviewdurchführung erfolgte telefonisch zwischen April und Mai 2020. Die Interviews dauerten zwischen 15 und 50 Minuten, wurden aufgezeichnet und wörtlich transkribiert. Die Datenauswertung erfolgte mit dem Programm ATLAS.ti. 8.1. Dazu wurden zufällig ausgewählte Interviews (etwa $25 \%$ des Materials) von 2 Autoren (MK, HS) unabhängig voneinander kodiert und zentrale Themenbereiche unter Anwendung einer induktiven Vorgehensweise identifiziert. Wiederkehrende thematische Aspekte wurden geclustert und im Rahmen eines iterativen Prozesses in der Forschungsgruppe hinsichtlich der Relevanz für die aufgestellten Fragen analysiert und zusammengeführt [16].

\section{Ergebnisse}

\section{Die Mitglieder unterstützten die Vereine auch während der Aussetzung des Trainings- und Wettkampfbetriebs}

Der Großteil der Interviewten berichtet, die Mitglieder hätten „verständnisvoll“, „mit großer Akzeptanz“ auf die Aussetzung des Trainings- und Wettkampfbetriebs reagiert. Vereinzelt sei es mit anhaltender Dauer des Lockdowns zu steigender Ungeduld und stärkerem Unmut gekommen.

[Die Reaktion war] neutral bis positiv. Die haben es alle verstanden, waren halt ein bisschen betrübt, weil sie gerne Sport machen wollen. Das ist halt eine Lebensaufgabe, [der Verein] ist Familie für viele (IP05, Vertreter Kampfsportverein).

Dementsprechend sei es nur vereinzelt zu Kündigungen bzw. Rückzahlungsforderungen gekommen. Begründet wurde das auch damit, dass die Vereine als solche auch als gesellschaftlich relevante Institutionen gesehen würden, die es auch in schwierigen Zeiten zu unterstützen gelte.

Ich habe angeboten, den Beitrag zu halbieren, was aber die meisten Mitglieder sogar abgelehnt haben, und [sie] gesagt haben: „Ja nee!“, dass sie quasi wollen, dass es weiterbesteht und [sie] weiter ...den vollen Beitrag zahlen werden (IP01, Vertreter Kampfsportverein).

Wir haben jetzt noch keine Austritte, ...aber sollte das länger dauern, kann das schon aufkommen, dass man sagt: „Wofür zahle ich hier die Beiträge?". ...Wobei wir natürlich kein Fitnessstudio sind, was nur gegen Training einen Eintritt ...hat, sondern wir sind auch ein gesellschaftlicher Verein, der ein Vereinsleben führt. Man sieht das hoffentlich auch von diesem Aspekt her (IP04, Vorstandsmitglied Judo- und Karateclub).
Vereine entwickeln oftmals ein breites Spektrum an z.T. interaktiven digitalen Kursangeboten, deren dauerhaftes Potenzial allerdings skeptisch betrachtet wird

Knapp 2/3 der Vereine haben während der Phase der Vereinsschließung digitale Trainingsformen entwickelt, um ihren Mitgliedern weiterhin ein Bewegungs- und Sportangebot zu unterbreiten. Dabei lassen sich 3 Angebotstypen unterscheiden: das Livestreaming von digitalen Trainingsangeboten, das digitale Zusenden von schriftlichen Trainingseinheiten sowie die Erstellung von Videos, die zeitunabhängig von Mitgliedern angeschaut werden können.

Es hat Abteilungen gegeben, die z. B. ein Cybertraining anbieten. So wie z. B. die Fußball-Abteilung. Da haben manche Mannschaften ... Onlinemeetings, wo der Trainer ... Sport macht oder was vorgibt und alle Spieler machen das nach... z. B. Stabi-Programm (IP15, Vertreter Mehrspartenverein).

Das einzige, was wir momentan anbieten, ist ein virtuelles Training für unsere Spieler ... die Spieler bekommen Übungen, Laufeinheiten, Krafteinheiten zugemailt, zugewhatsappt, damit sie einen gewissen Fitnessgrad behalten (IP03, Vorstandsmitglied Handballsportverein).

Ich habe das in der Kindersportschule so gemacht, dass wir wöchentlich ein kleines Video machen von den Übungsleitern. ... Das Video besteht immer aus einem turnerischen Element und einem koordinativen Element... das stellen wir auf Facebook, es können alle anschauen, ....so ein Hometraining, wo es die Kinder dann zuhause ausprobieren können (IP15, Vertreter Mehrspartenverein).

Vereinzelt wurde versucht, über die virtuellen Plattformen auch den Kontakt und Austausch in den jeweiligen Gruppen zu erreichen. Dazu konnten Mitglieder Videos von sich selber für andere hochladen. Gerade bei Kindern wurden darüber hinaus zusätzliche Anstrengungen unternommen, um sie zur Nutzung der digitalen Formate zu motivieren.

Es gibt von der ersten Männermannschaft und ersten Damenmannschaft über Instagram so ein paar witzige Videos, die sie reinstellen, damit man visuell in Kontakt bleibt ... posten, wie sie irgendwelche Übungen in einer witzigen Verkleidung ausführen (IP03, Vorstandsmitglied Handballsportverein).

[Ein] paar Trainer von Jugendmannschaften [machen] immer so Challenges ... die Kinder müssen aufgezeichnet werden und [das Video] dem Trainer schicken, dann wird's bewertet mit Punkten, und dann ... wird das ausgewertet und gibt's kleine Überraschung..., dass der Ansporn noch ansteigt (IP02, Vorstandsmitglied Mehrspartenverein).

Bei den Angeboten virtueller Trainings lässt sich kein eindeutiger Trend zwischen Ein- und Mehrspartenvereinen erkennen. Von vielen Befragten wurde berichtet, dass die digitalen Sportangebote in Eigeninitiative von einzelnen Übungsleiterinnen und Übungsleitern erstellt und nicht systematisch vom Vorstand aus geplant und beauftragt wurden. 
[Bereitstellung virtueller Angebote] ... waren Eigeninitiativen der Abteilungen, die sich überlegt haben: „wie können wir an unsere Mitglieder ran?“... da ist unser Verein zu groß, zu vielfältig, dass [wir] das überblicken können vom Vorstand (IP14, Vorstandsmitglied Mehrspartenverein).

In einzelnen Vereinen wurde der Zugang zu den digitalen Sportangeboten öffentlich zugänglich gemacht, um damit das eigene Angebot auch bei Nicht-Vereinsmitgliedern bekannt zu machen.

Der Link ... steht bei uns auf der Homepage, da kann sich im Prinzip jeder anmelden. Wir haben es ... öffentlich [gemacht], da machen wir keinen Unterschied... Ist ja für uns nicht mehr Aufwand ... Ist auch eine Werbung sozusagen (IP11, Vorstandsmitglied Skiclub).

Trotzdem wird von der Mehrheit der befragten Personen beschrieben, dass die digitalen Bewegungs- und Sportangebote das persönliche Training nicht (dauerhaft) ersetzen könnten. Dafür wurden unterschiedliche Gründe genannt, die in nachfolgender $>$ Tab. 1 weiter ausgeführt werden.

Wenige Vereinsvertreter berichten, dass die Bereitstellung ihres Online-Angebots zukünftig beibehalten und z.T. ausgebaut werden soll, um neue Mitglieder zu gewinnen, bzw. um wettbewerbsfähig zu bleiben.

[Das digitale Angebot für die Zukunft beizubehalten], kann ich mir grundsätzlich vorstellen, das müsste man aber besprechen ... auch mit den Trainern, ob sie das aufrechterhalten wollen...lch würde sagen, dass wir über diese Online-Trainingsangebote neue Mitglieder finden. ... Das sehe ich auch als Werbung für uns (IP11, Vorstandsmitglied Skiclub).

\section{Resonanz der Vereinsmitglieder auf das veränderte Sportangebot}

Die Befragten berichten überwiegend positiv von der Nutzung der bereitgestellten digitalen Formate, können aber das Ausmaß der Teilnahme an den Online-Kursangeboten insgesamt nur schwer beurteilen. Bei den Live-Angeboten wird beobachtet, dass z. T. weniger, z. T. aber auch mehr Personen trainieren als bei den Präsenzformaten. Dabei lässt sich bezüglich der Gruppen, die laut der Befragten bevorzugt die digitalen Formate nutzten, kein Muster oder kein einheitlicher Trend erkennen.

[Akzeptanz ist] relativ spärlich. Das Mitmachen ist ungefähr ein Drittel von den Leuten, die sonst in einem normalen Training sind ... Was man so hört: „Alles wird digital und Generation Y“, die haben an den Trainings nicht teilgenommen, sondern das waren dann eher ...die Älteren irgendwo zwischen 35, 40 (IP01, Vertreter Kampfsportverein).

[Nutzer der Online-Angebote] ... das sind lustigerweise wesentlich mehr als die, die normalerweise ins Hallentraining kommen zu uns. Da sind auch Teilnehmer aus befreundeten anderen Vereinen dabei (IP11, Vorstandsmitglied Skiclub).
Wobei im regulären Hallentraining noch mehr Ältere dabei sind, da haben wir eine spezielle Gruppe, wo eben der Altersdurchschnitt sehr hoch ist. (IP 11, Vorstandsmitglied Skiverein) Von [den] Videos würde ich sagen, dass wahrscheinlich rund die Hälfte der Mitglieder diese Trainings gemacht hat, von den Kindern v. a. Bei den Erwachsenen viel weniger (IP01, Vertreter Kampfsportverein).

\section{Vereine entwickeln aufwendige und flexible Konzepte, um den Trainingsbetrieb schrittweise wieder starten zu können}

Die Erarbeitung von Konzepten zur Wiederaufnahme des Sportbetriebs erfordert einerseits die Auseinandersetzung mit gesetzlichen Vorgaben, Empfehlungen von Fachverbänden und dem Bayrischen Landes-Sportverband e.V. (BLSV), Dachorganisation des Vereinssports in Bayern, sowie andererseits kreative Ideen, die ein kontaktfreies Training ermöglichen. Es werden verschiedene Planungsszenarien und Sicherheitskonzepte erarbeitet. Erschwerend kommt hinzu, dass sich die Vorgaben ständig ändern würden.

Ich hab jetzt schon einige Zeit verbracht mit Studieren von Vorschlägen von Fachverbänden, vom BLSV, gesetzlichen Vorgaben, um irgendwie zu einem stimmigen Konzept zu kommen, wie wir es umsetzen können, ohne gegen Hygiene oder sonstige Vorschriften zu verstoßen (IP14, Vorstandsmitglied Mehrspartenverein).

Dass man ganz gezielt nur die Trainingsinhalte trainieren lässt, wo keine Nähe vorhanden ist, also keine Zweikampfübungen, ... nur das trainieren, wo wir keine Berührung mit Haut, mit Spucken, Tröpfcheninfektion, ...dass über die Distanz sichergestellt werden kann, dass keine Übertragung stattfindet. Und da gibt es ganz, ganz viele Übungen, die man machen kann. Von Ballkontrolle, Ballgefühl kann man in fünf Reihen parallel nebeneinander ausführen lassen, ohne dass die Kinder nah bei einander sind. Wir haben vier Fußballplätze, ... die Kinder wären weit genug auseinander (IP07, Vertreter Mehrspartenverein).

\section{Diskussion}

Die Corona-bedingten Einschränkungen im Sportvereinsbetrieb haben gemäß der befragten Personen nicht zu einem bedeutsamen Mitgliederrückgang geführt. Vielmehr würden die Vereinsmitglieder die Sportvereine als wichtige gesellschaftliche Institution unterstützen wollen. Außerdem wurden laut den Interviews umfangreiche Aktivitäten entwickelt, um den Mitgliedern dennoch ein geregeltes Sportangebot (z. B. in digitaler Form) zu unterbreiten sowie Kontakt und Austausch zwischen den Mitgliedern zu fördern. In welchem Umfang und in welcher Form alternative Angebote entwickelt wurden, hing dabei in erster Linie von der Eigeninitiative einzelner Übungsleiterinnen und Übungsleiter ab. Die Nutzung von digitalen Angeboten durch die Mitglieder scheint sich von der Nutzung der realen Kurse zu unterscheiden. Aus den Interview-Antworten lässt sich schließen, dass damit verschiedene Zielgruppen unterschiedlich gut erreicht wurden. Es hat sich hier aber kein einheitliches Muster bei den befragten Vereinen gezeigt. Während vereinzelt Vereinsvertreter planen, digitale Angebote v. a. zur 
• Tab. 1 Begründungen, warum digitale Sportangebote das persönliche Training nicht (dauerhaft) ersetzen können.

\begin{tabular}{|c|c|}
\hline Begründung & Beispielzitate \\
\hline $\begin{array}{l}\text { Die Teilnehmenden würden insbesondere } \\
\text { in Mannschaftssportarten den Kontakt mit } \\
\text { anderen mögen und bräuchten den } \\
\text { Gemeinschaftsaspekt beim Sport. }\end{array}$ & $\begin{array}{l}\text { Die Menschen, die Handball spielen, haben } 3 \text { Grundeigenschaften. 1. Sie wollen einen Ball. 2. Sie suchen } \\
\text { Körperkontakt. 3. Sie suchen die Gemeinschaft. Und das ist [mit Videos] alles nicht durchführbar. Natürlich } \\
\text { schaut man sich jetzt auch mal Videos an ... aber der junge Mensch, der Handball spielt, braucht den Ball, den } \\
\text { Kontakt und die Gemeinschaft. Und das geht virtuell nicht. Auf Dauer, auf gar keinen Fall (IP03, Vorstandsmit- } \\
\text { glied Handballsportverein). } \\
\text { Wenn man Mannschaftssportarten nimmt, [ist das] überhaupt nicht sinnvoll, das [digital] zu machen, weil ich } \\
\text { eben auf Mannschaftstraining angewiesen bin. ... Da geht's in den meisten Fällen darum, dass die zusammen- } \\
\text { kommen wollen, miteinander sprechen ... Also dass da das Zusammensein eher wichtiger ist (IP14, Vorstands- } \\
\text { mitglied Mehrspartenverein). }\end{array}$ \\
\hline $\begin{array}{l}\text { Manche Sportarten ließen sich nur in spezi- } \\
\text { fischen Sportstätten lernen und praktizie- } \\
\text { ren, oder nur in Gruppen, v. a. Kontakt- } \\
\text { oder Mannschaftssport. }\end{array}$ & $\begin{array}{l}\text { Aber jetzt theoretisch schwimmen lernen oder zu schwimmen, das macht keinen Sinn (IP08, Vorstandsmitglied } \\
\text { Schwimmverein). } \\
\text { Das Spiel miteinander, auch die Zweikämpfe, auch gewisse Spielkombinationen, das kann man so halt nicht } \\
\text { trainieren. Da kann man individuell trainieren, Technik, Dribbelverhalten, Koordination, Ausdauer, aber das } \\
\text { Spiel an sich, das kann man halt auch nur gemeinsam am Platz trainieren (IP07, Vertreter Mehrspartenverein). }\end{array}$ \\
\hline $\begin{array}{l}\text { Manche Sportarten würden die persönli- } \\
\text { che Anleitung, direkte Kontrolle und } \\
\text { Korrektur vom Trainer benötigen. }\end{array}$ & $\begin{array}{l}\text { Im normalen Training [kann] ich ... mich neben einem Einzelnen hinstellen und ... zeigen, was er besser machen } \\
\text { kann. Ich kann den berühren, ich kann dem seine Hand führen, seinen Fuß mal halten usw., und das kann ich } \\
\text { online nicht (IP01, Vertreter Kampfsportverein). }\end{array}$ \\
\hline $\begin{array}{l}\text { Insbesondere Kinder ließen sich im } \\
\text { persönlichen Kontakt und Spiel besser an } \\
\text { den Sport heranführen. }\end{array}$ & $\begin{array}{l}\text { In der Kindersportschule ist es so, wir leben von dem Kontakt mit den Kindern. Dass man den Kindern das } \\
\text { vermittelt, dass man die in die Sportarten einführt ... Das ist auf jeden Fall nicht möglich in der Kindersport- } \\
\text { schule, dass man das online weitermacht (IP15, Vertreter Mehrspartenverein). }\end{array}$ \\
\hline $\begin{array}{l}\text { Bei manchen Vereinsmitgliedern könnte } \\
\text { unzureichendes IT-Verständnis die } \\
\text { Teilnahme an digitalen Sportangeboten } \\
\text { erschweren. }\end{array}$ & $\begin{array}{l}\text { In dem Präventions-Bereich [sind] viele Ältere, wo ich nicht glaube, dass uns die alle digital folgen können. Dass } \\
\text { das vielleicht der ein oder andere gut 70-jährige schafft, hier über Skype ... teilzunehmen, aber ich glaube, die } \\
\text { Mehrheit von diesen Teilnehmern wird wohl nicht in der Lage sein, das technisch mit hinzubringen (IP08, } \\
\text { Vorstandsmitglied Schwimmverein). }\end{array}$ \\
\hline $\begin{array}{l}\text { Es könnten sich technische und daten- } \\
\text { schutzrechtliche Probleme ergeben. }\end{array}$ & $\begin{array}{l}\text { Mit WhatsApp ganz schwierig. WhatsApp ruckelt, WhatsApp nimmt viel Speicher in Anspruch auf dem Handy } \\
\text { (IP07, Vertreter Mehrspartenverein). } \\
\text { Die ...Möglichkeit wäre ja, einfach das normale Training zu streamen, aber da hat man das Problem mit } \\
\text { Datenschutz. Dass einige Mitglieder natürlich nicht wollen, dass sie vielleicht beim Training online beobachtet } \\
\text { werden usw. Deswegen glaube ich eher nicht, dass ich das machen werde (IP01, Vertreter Kampfsportverein). }\end{array}$ \\
\hline
\end{tabular}

Mitgliederwerbung auch langfristig fortzuführen, sehen die meisten Befragten das längerfristige Potential skeptisch. Insbesondere der Gemeinschaftsaspekt und der körperliche Kontakt, z. B. bei Mannschaftssportarten und bei der Anleitung durch die Übungsleiterinnen und Überleiter, wären essentielle Elemente des Sports, die im digitalen Format fehlen würden. Schließlich wurden erhebliche Anstrengungen berichtet, um der Dynamik der Pandemie Rechnung zu tragen und die Bewegungsangebote schrittweise den sich ändernden Distanz- und Schutzgeboten anzupassen.

\section{Vergleich mit anderen Studien}

Bisherige Publikationen zum Thema befassen sich in erster Linie damit, wie auch unter Pandemiebedingungen (professionelle) Sportwettkämpfe und -veranstaltungen wieder durchgeführt werden können (vgl. z. B. [17, 18] . Nach unserer besten Kenntnis wurden die Auswirkungen der Eindämmungsmaßnahmen auf Anbieter im Breitensport und damit auf Public Health bislang weltweit noch nicht untersucht.

\section{Stärken und Limitationen}

Es handelt sich um die erste Studie, in der die COVID-19-bedingten Konsequenzen für Sportvereine erfasst wurden. Die Ergebnisse dieser explorativen Studie in einer Stichprobe von 15 Sportvereinen können nicht als repräsentativ für die Gesamtheit der Sportvereine gewertet werden. Das ist jedoch auch nicht der Anspruch qualitativer Datenerhebungen. Vielmehr geht es darum, das Spektrum möglicher Verän- derungen und sozialer Prozesse auszuloten und Zusammenhänge zu verstehen. Durch die bewusste Einbeziehung verschiedener Sportvereinsarten, insbesondere von Ein- und Mehrspartenvereinen mit verschiedenen Sportarten, wurden unterschiedliche mögliche Einflussfaktoren berücksichtigt. Es handelt sich allerdings um eine Studie in einem Bundesland (Bayern); die Veränderungen können in anderen Bundesländern unterschiedlich ausfallen. Es zeigte sich zudem, dass die Nutzung der digitalen Angebote durch Vereinsmitglieder von den Befragten oft nur geschätzt werden konnte, da die Nutzungshäufigkeit nicht regelhaft erfasst wurde; diese Daten wären durch Mitgliederbefragungen besser zu erfassen. Dennoch erscheint es wichtig, die Eindrücke der Befragten zu erfassen, da sie für deren Bewertung (und ggf. Fortführung) digitaler Angebote wichtig sind.

\section{Implikationen für Politik und Praxis}

Die Ergebnisse zeigen, dass Sportvereine für viele Mitglieder eine Institution darstellen, mit der sie sich identifizieren und die sie auch dann unterstützen, wenn es vorübergehend zu Angebotseinschränkungen kommt. Die Vereine haben darüber hinaus schnell und flexibel auf die neuen Vorgaben reagiert und sich bemüht, den Mitgliedern alternative (digitale) Bewegungs- und Sportangebote zu unterbreiten. Diese Befunde unterstreichen die Bedeutung von Sportvereinen für den Breitensport und auch für die bevölkerungsbezogene Bewegungsförderung während der COVID-19 Pandemie. Studien deuten darauf hin, dass es während des Lockdowns zu einem Rückgang der körperlichen Aktivität bei vielen Menschen 
kam (vgl. z. B. [19-21]), wenngleich die Ergebnisse nicht eindeutig sind [22]. Aus den Interviews wurde darüber hinaus deutlich, dass Sportvereine von den Mitgliedern insbesondere auch als soziale und gemeinschaftsfördernde Institution geschätzt werden. Dementsprechend ist es bis zum Zeitpunkt der Interviews auch kaum zu Kündigungen oder Beitragsrückforderungen gekommen. Naturgemäß konnte der Wunsch nach Kontakt und Gemeinschaft bei bestehenden sozialen Distanzmaßnahmen nicht vollumfänglich erfüllt werden. Doch die Verantwortlichen haben kreative Wege gefunden, über interaktive und spielerisch-kompetitive Elemente die Bindung und das Gemeinschaftsgefühl aufrechtzuerhalten. Die Vereine können damit auch einen Beitrag zur psychosozialen Gesundheitsförderung leisten und negativen Entwicklungen, wie sie während der Corona-bedingten Einschränkungen aufgetreten sind [23], vorbeugen. Der Stellenwert der Vereine als wichtiger Kooperationspartner für lokale Gesundheitsförderungsmaßnahmen bleibt damit - gerade auch unter den Rahmenbedingungen einer Pandemie - ungebrochen hoch.

Trotz der grundsätzlich positiven Erfahrungen zeigte sich die Mehrheit der Befragten skeptisch, was das Potenzial digitaler Angebote für das zukünftige Vereinsprogramm betrifft. Hierzu erscheint zusätzliche Forschung notwendig, da aus den Interviews auch deutlich wurde, dass durch die digitalen Angebote teilweise andere bzw. neue Zielgruppen angesprochen wurden. Darüber hinaus lehnten viele ältere Personen aufgrund eines Infektionsrisikos die Teilnahmen an realen Kursen ab [24]. Möglicherweise bietet die Kombination von digitalen mit realen Angeboten die Möglichkeit, verschiedene Bevölkerungsgruppen anzusprechen und somit soziale Ungleichheiten beim Zugang zu Bewegungs- und Gesundheitsförderung zu reduzieren $[25,26]$. Zukünftige Studien sollten daher stärker die Nutzung und Akzeptanz digitaler Sportangebote unter Vereinsmitgliedern erfassen. Des Weiteren sollten Wissensaustauschprozesse [27] zwischen den Vereinen untereinander sowie mit Public-Health-Experten und Expertinnen zu besonders erfolgreichen digitalen Formaten und/oder Trainingskonzepten initiiert werden, um den Vereinen eine effizientere Umsetzung digitaler Angebote zu ermöglichen. Schließlich sollte in zukünftigen Studien untersucht werden, in welcher Form das Infektionsrisiko und die Auswirkungen einer Covid-19 Infektion im Vereinssport (Quarantäne-Anordnung) die Teilnahmebereitschaft und Wiederaufnahme von Bewegungs- und Sportangeboten in Präsenzform beeinflussen.

\section{Förderung}

Die Studie fand im Rahmen des BMBF-geförderten Forschungsprojektes ACTION for men (A4M) statt, als Teil des Forschungsverbundes Capital4Health (Fkz: 01EL1421A) und wird gemeinsam von den Universitäten Regensburg (Fkz: 01EL1421H) sowie Bayreuth (Fkz: 01EL1421D) durchgeführt.

\section{Interessenkonflikt}

Die Autorinnen/Autoren geben an, dass kein Interessenkonflikt besteht.
Literatur

[1] Deutscher Olympischer Sportbund. Bestandserhebung 2019, 2020; https://cdn.dosb.de/user_upload/www.dosb.de/medien/BE/ BE-Heft_2019.pdf

[2] Breuer C. Sportentwicklungsbericht 2015/2016. Analyse zur Situation der Sportvereine in Deutschland. Bonn: Bundesinstitut für Sportwissenschaft; 2017

[3] Hilbert J, Enste P. Aktive „silberne Mitarbeit“statt passive „goldene Vereinsnadel" - Gesellschaftliche Potenziale in Sportvereinen nutzen!. Public Health Forum 2007; 15: 16-18. doi:10.1016/j.phf.2007.10.010

[4] Breuer C, Feiler S. Sportvereine in Deutschland: Organisationen und Personen. Sportentwicklungsbericht für Deutschland 2017/2018 - Teil 1. Bonn: Bundesinstitut für Sportwissenschaft; 2019

[5] Bös K, Brehm W. editor Handbuch Gesundheitssport. Schorndorf: Hofmann; 2006

[6] Kokko S, Martin L, Geidne S et al. Does sports club participation contribute to physical activity among children and adolescents? A comparison across six European countries. Scandinavian Journal of Public Health 2019; 47: 851-858. doi:10.1177/1403494818786110

[7] Jekauc D, Reimers AK, Wagner MO et al. Physical activity in sports clubs of children and adolescents in Germany: results from a nationwide representative survey. Journal of Health Monitoring 2013; 21: 505-513. doi:10.1007/s10389-013-0579-2

[8] Neuber N. editor. Informelles Lernen im Sport: Beiträge zur allgemeinen Bildungsdebatte. $1^{\text {st }}$ ed. Wiesbaden: VS Verl. für Sozialwiss; 2010

[9] Burrmann U, Mutz M, Zender U. editors. Jugend, Migration und Sport: Kulturelle Unterschiede und die Sozialisation zum Vereinssport. Wiesbaden: Springer VS; 2015

[10] Strobl H, Brew-Sam N, Curbach J et al. ACTION for Men: Study Protocol of a Community Capacity Building Intervention to Develop and Implement Gender-Sensitive Physical Activity Programs for Men 50 Plus. Front Public Health 2020; 8: 4

[11] Loss J, Brew-Sam N, Metz B et al. Capacity Building in Community Stakeholder Groups for Increasing Physical Activity: Results of a Qualitative Study in Two German Communities. Int J Environ Res Public Health. 2020; 17

[12] Deutsche Bundesregierung. Erweiterung der beschlossenen Leitlinien zur Beschränkung sozialer Kontakte 2020; https://www.bundesregierung.de/breg-de/themen/coronavirus/besprechung-der-bundeskanzlerin-mit-den-regierungschefinnen-und-regierungschefs-der-laender- 1733248

[13] Deutsche Bundesregierung. Leitlinien gegen Ausbreitung des Coronavirus 2020; https://www.bundesregierung.de/breg-de/ themen/coronavirus/leitlinien-bund-laender-1731000

[14] Deutsche Bundesregierung. 6. Mai 2020: Regeln zum Corona-Virus. 2020; https://www.bundesregierung.de/breg-de/leichte-sprache/6mai-2020-regeln-zum-corona-virus-1755252

[15] Helfferich C. Die Qualität qualitativer Daten: Manual für die Durchführung qualitativer Interviews. $4^{\text {th }}$ ed. Wiesbaden: VS Verlag für Sozialwissenschaften; 2011

[16] Ritchie J, Lewis J.editors Qualitative research practice: A guide for social science students and researchers. $2^{\text {nd }}$ ed. London: SAGE; 2014

[17] Herrero-Gonzalez H, Martín-Acero R, Del Coso J et al. Position statement of the Royal Spanish Football Federation for the resumption of football activities after the COVID-19 pandemic (June 2020). British journal of sports medicine. 2020; doi:10.1136/bjsports-2020-102640

[18] Carmody S, Murray A, Borodina M et al. When can professional sport recommence safely during the COVID-19 pandemic? Risk assessment and factors to consider. British journal of sports medicine 2020; 54 : 946-948. doi:10.1136/bjsports-2020-102539 
[19] Ammar A, Brach M, Trabelsi K et al. Effects of COVID-19 Home Confinement on Eating Behaviour and Physical Activity: Results of the ECLB-COVID19 International Online Survey. Nutrients 2020; doi:10.3390/nu12061583

[20] Stanton R, To QG, Khalesi S et al. Depression, Anxiety and Stress during COVID-19: Associations with Changes in Physical Activity, Sleep, Tobacco and Alcohol Use in Australian Adults. Int J Environ Res Public Health 2020; doi:10.3390/ijerph17114065

[21] Qin F, Song Y, Nassis GP et al. Physical Activity, Screen Time, and Emotional Well-Being during the 2019 Novel Coronavirus Outbreak in China. Int J Environ Res Public Health 2020; doi:10.3390/ ijerph17145170

[22] Jordan S, Krug S, Manz K et al. Gesundheitsverhalten und COVID-19: Erste Erkenntnisse zur Pandemie. Journal of Health Monitoring 2020; 5: 1-16. doi:10.25646/7054

[23] Maugeri G, Castrogiovanni P, Battaglia G et al. The impact of physical activity on psychological health during Covid-19 pandemic in Italy. Heliyon. 2020; 6: e04315. doi:10.1016/j.heliyon.2020.e04315
[24] Goethals L, Barth N, Guyot J et al. Impact of Home Quarantine on Physical Activity Among Older Adults Living at Home During the COVID-19 Pandemic: Qualitative Interview Study. JMIR Aging. 2020; 3: e19007. doi:10.2196/19007

[25] Abel T, Frohlich KL. Capitals and capabilities: linking structure and agency to reduce health inequalities. Social Science \&. Medicine. 2012; 74: 236-244. doi:10.1016/j.socscimed.2011.10.028

[26] Strobl H. Theoretical underpinnings of interventions that effectively promote physical activity in adult men. Ger J Exerc Sport Res 2019; 4: e78

[27] Rütten A, Frahsa A, Abel T et al. Co-producing active lifestyles as whole-system-approach: theory, intervention and knowledge-toaction implications. Health Promot Int 2019; 34: 47-59. doi:10.1093/ heapro/dax053 